\title{
Enhanced Montgomery Multiplication
}

\author{
Shay Gueron ${ }^{1,2}$ \\ 1 Department of Mathematics, University of Haifa, Haifa 31905, Israel \\ 2 Discretix Technologies, Netanya, Israel \\ shay@math.haifa.ac.il
}

\begin{abstract}
We define the Non Reduced Montgomery Multiplication of order $s$, of $A$ and $B$, modulo $N$ (odd integer) by $N R M M^{s}(A, B, N)=$ $\left(A B+N\left(-A B N^{-1} \quad\left(\bmod 2^{s}\right)\right)\right) 2^{-s}$. Given an upper bound on $A$ and $B$, with respect to $N$, we show how to choose the variable $s$ in a way that guarantees that $N R M M^{s}(A, B, N)<2 N$.

A few applications are demonstrated, showing the advantage of using $N R M M^{s}$ with an appropriately chosen $s$, over the classical Montgomery Multiplication.
\end{abstract}

\section{Introduction}

Various public key cryptosystems are based on computing modular arithmetic functions (e.g., modular exponentiation and modular multiplication). Thus, methods for fast computation of such operations, particularly in hardware, are of great importance for practical cryptography. One of the most successful methods known today, whose advantage is mostly appreciated in hardware realization, is the Montgomery multiplication [2]. As such, it is a standard approach in hardware implementations of, for example, RSA, digital signature schemes, Diffie-Hellman key exchange, and elliptic curve cryptography (over $G F(p)$ ).

Despite its advantage, the Montgomery multiplication method suffers from a drawback: the need to reduce the final result to $[0, N)$. This complicates the related implementation and also creates a security problem.

In this paper, we show how to overcome this drawback by using the Non Reduced Montgomery Multiplication of order $s$, where $s$ is appropriately chosen according to the application at hand. The described method is a generalization of modifications to the Montgomery algorithm (e.g., 4], [1]).

\section{Preliminaries and Definitions}

Definition 1 (Non Reduced Montgomery Multiplication of order $s$ $\left.\left(N R M M^{s}\right)\right)$.

Let $A, B$ and s be positive integers, and let $N$ be an odd positive integer. The Non Reduced Montgomery Multiplication of order s, of $A, B$, modulo $N$ is defined by

$$
N R M M^{s}=N R M M^{s}(A, B, N)=\frac{A B+N\left(-A B N^{-1}\left(\bmod 2^{s}\right)\right)}{2^{s}}
$$


Remarks: By looking at the quantity $A B+N\left(-A B N^{-1} \quad\left(\bmod 2^{s}\right)\right)$ modulo $2^{s}$, it is clear that $\operatorname{NRMM}^{s}(A, B, N)$ is an integer. Also, considering $A B+$ $N\left(-A B N^{-1}\left(\bmod 2^{s}\right)\right)$ modulo $N$, we can conclude that $N R M M^{s}(A, B, N)$ $(\bmod N)=A B 2^{-s}(\bmod N)$. On the other hand, even when $A, B<N$, the value of $N R M M^{s}(A, B, N)$ is not necessarily reduced modulo $N$ (i.e., it is not necessarily in the range $[0, N))$. An example for input $A, B<N$ where $\operatorname{NRMM}^{s}(A, B, N)$ is nonreduced is $\operatorname{NRMM}^{5}(17,18,19)=25>19$.

Algorithm 1 below describes the bit-level computation of $N R M M^{s}(A, B, N)$, for $s$ such that $s \geq 1+\left[\log _{2} A\right]$. For this description, we denote the binary representation of a number $Q$ by a string $Q=\left[Q_{m-1} \ldots Q_{1} Q_{0}\right]$ of $m$ bits, where $m=1+\left[\log _{2} Q\right]$, and where $Q_{0}$ is the least significant bit of $Q$.

Algorithm 1: (Non Reduced Montgomery Multiplication of order $s$ )

Input: $A, B, N(N$ is odd $), s\left(s \geq 1+\left[\log _{2} A\right]\right)$

Output: $N R M M^{s}(A, B, N)$

$S=0$

For $i$ from 0 to $s-1$ do

$\begin{array}{ll}1.1 & S=S+A_{i} B \\ 1.2 & S=S+S_{0} N \\ 1.3 & S=S / 2\end{array}$

End For

Return $S$

Note that although by definition, $N R M M^{s}$ is symmetric in $A$ and $B$, this is not the case its bit-level implementation, in particular when $A$ and $B$ have a different number of bits in their binary representation.

It is not difficult to see that the output of Algorithm 1 is $N R M M^{s}(A, B, N)$, as we now explain. Steps 1.1, 1.3 compute (bit by bit) the quantity $A B 2^{-s}$, where step 1.2 is a "parity correction": the odd number $N$ is conditionally added to $S$ whenever it is odd and cannot be straightforwardly divided by 2 (in the subsequent step 1.3). Since $s \geq 1+\left[\log _{2} A\right]$, all the bits of $A$ are scanned. Thus, Algorithm 1 actually computes $(A B+K N) / 2^{s}$ for some $K$. The number $K$ is constructed (bit by bit, in step 1.2) in a way that $2^{s} \mid(A B+K N)$. Since $K$ has altogether $s$ bits, $K<2^{s}$. The only value of $K<2^{s}$ satisfying $A B+K N \equiv 0$ $\left(\bmod 2^{s}\right)$ is $K=-A B N^{-1}\left(\bmod 2^{s}\right)$.

Relation to the classical Montgomery multiplication. The classical Montgomery multiplication of $A$ and $B$ modulo $N$, under the condition $A, B<N$, is defined as $M M U L(A, B, N)=A B 2^{-n}(\bmod N)$, where $n=1+\left[\log _{2} N\right]$. Thus, $M M U L(A, B, N)$ can be viewed as the special case of $N R M M^{s}(A, B, N)$ where $s=1+\left[\log _{2} N\right]=n$, but with a reduced result, that is: $M M U L(A, B, N)=$ $N R M M^{n}(A, B, N) \quad(\bmod N)$.

Observing Algorithm 1 for $A, B<N$, it can be shown that $S<2 N$ after step 1.3. The proof is by induction. Starting with $S<2 N$ before step 1.1 (the algorithm is initialized with $S=0), S$ can increase in steps 1.1-1.3 up to $(S+$ 
$B+N) / 2<(2 N+N+N) / 2=2 N$. Since $S<2 N$ in any step of Algorithm 1, we can deduce a bit level algorithm for computing $M M U L(A, B, N)$ (which reduces to the classical algorithm for computing the Montgomery Multiplication): it is the same as Algorithm 1 with the particular choice $s=n$, followed by a single reduction step (if $S>N$ then $S=S-N$ ) after the end of the For-loop. Algorithm 2 describes this procedure.

Algorithm 2: (Classical Montgomery Multiplication)

Input: $A, B, N\left(A, B<N, n=1+\left[\log _{2} N\right], N\right.$ is odd $)$

Output: $M M U L(A, B, N)$

$S=0$

For $i$ from 0 to $n-1$ do

$2.1 \quad S=S+A_{i} B$

$2.2 \quad S=S+S_{0} N$

$2.3 \quad S=S / 2$

End For

2.4 if $S>N$ then $S=S-N$

Return $S$

Note that unlike Algorithm 1, Algorithm 2 is symmetric in $A$ and $B$.

The main advantage in using the Montgomery multiplication lies in the hardware implementation of Algorithm 2: it requires only additions and divisions by 2. More important is the special property of step 2.2: only the least significant bit of the cumulative result $(S)$ must be known in order to determine whether or not $N$ should be added, and to carry on to the next step. In fact, this is the key feature that makes Montgomery multiplication superior to standard modular multiplication algorithms, and therefore what made it a de-facto standard in cryptographic hardware.

The Montgomery multiplication is especially efficient when a sequence of MMUL operations is used in a row. For example, consider the task of computing modular exponent $A^{E} \quad(\bmod N)(A<N)$ which can be carried out as follows. The constant $H=2^{2 n}(\bmod N)$ (where $\left.n=1+\left[\log _{2} N\right]\right)$ is pre-computed. The input $A$ is transformed to what is called the Montgomery domain (or Montgomery base): $A^{\prime}=A 2^{n}(\bmod N)=M M U L(A, H, N)$. Then, the square and multiply (or any other) exponentiation algorithm is applied to $A^{\prime}$ with the following adaptation that accounts for being in the Montgomery domain: Montgomery multiplications are used instead of regular modular multiplications. The end result is transformed back to the real domain by Montgomery multiplication by 1 (since for any $X$, we have $\left.M M U L\left(X^{\prime}, 1, N\right)=X \quad(\bmod N)\right)$. These steps are summarized in Algorithm 3.

Algorithm 3: (Modular exponentiation via Montgomery Multiplication) Input: $A, E, N(N$ is odd, $A<N), n=1+\left[\log _{2} N\right], m=1+\left[\log _{2} E\right], H=2^{2 n}$ $(\bmod N)$

Output: $A^{E} \quad(\bmod N)$

Initialization: $A^{\prime}=M M U L(A, H, N)=A 2^{n} \quad(\bmod N) ; T_{m-1}=A^{\prime}$

$S=0$ 
For $i$ from $m-2$ to 0 do

$3.1 \quad T_{i}=\operatorname{MMUL}\left(T_{i+1}, T_{i+1}, N\right)$

$3.2 \quad$ if $E_{i}=1$ then $T_{i}=\operatorname{MMUL}\left(T_{i}, A^{\prime}, N\right)$

End For

$3.3 T_{0}=M M U L\left(T_{0}, 1, N\right)$

Return $T_{0}$

The advantage of Algorithm 3 is that expensive modular multiplications in the real domain are replaced by the analogous cheap (hardware wise) Montgomery multiplications in the Montgomery domain. The overhead of the transformation from and to the real domain is insubstantial: the cost is only two additional Montgomery multiplications (by $H$ at the beginning and by 1 at the end).

It is important to note that the reduction step 2.4 is crucial. Without reduction, the output of one $M M U L$ operation is not necessarily a legal input to the subsequent $M M U L$.

Unfortunately, the need for reduction in each $M M U L$ operation substantially complicates hardware realization of Algorithm 2. Dedicated circuitry is required for detecting the cases where the result is greater than $N$, and for performing the appropriate subtraction. Furthermore, this conditional subtraction exposes the implementation to side channel attacks. Consequently, although the Montgomery multiplication enables efficient hardware implementation of modular arithmetic operations, a method that does not require repeated reductions can be a significant improvement. We show here that the use of $N R M M^{s}$, together with a proper choice of the parameter $s$, is a proper solution.

\section{The Appropriate Choice of $s$ in the $N R M M^{s}$ Algorithm}

The following Lemma shows that the output of Algorithm 1 can be made smaller than $2 N$ for input $A$ and $B$ of any size, provided that the parameter $s$ is properly chosen. It also determines the required size of the accumulator $S$, when Algorithm 1 is used.

\section{Lemma 1.}

Let $N$ be a given positive odd number, and $n=1+\left[\log _{2} N\right]$. Let $A$, and $B$ be nonnegative integers such that $A \leq 2^{k} N$ and $B \leq 2^{k} N$, for some $k \geq 0$. Let $s$ be a positive integer such that $s \geq n+2 k$. Then,

a) $N R M M^{s}(A, B, N)<2 N$.

b) $s=n+2 k$ is the smallest integer that guarantees that $N R M M^{s}(A, B, N)<$ $2 N$.

c) $N R M M^{s}(A, 1, N) \leq N$ with equality only if $A$ is a multiple of $N$.

d) When applying Algorithm 1, the accumulator $S$ is bounded by $2\left(1+2^{k}\right) N$. Thus, the accumulator should be capable of storing $n+k+2$ bits. 
Proof.

(a) Take $s \geq n+2 k$. By definition, $N<2^{n}$. Using this bound, we have

$$
\begin{gathered}
N \operatorname{RM}^{s}(A, B, N)=\frac{A B+N\left(-A B N^{-1}\left(\bmod 2^{s}\right)\right)}{2^{s}} \leq \\
\frac{2^{2 k} N^{2}+N\left(2^{s}-1\right)}{2^{n+2 k}}=N\left(\frac{N}{2^{n}}+\frac{2^{s}-1}{2^{s}}\right)<2 N
\end{gathered}
$$

(b) To show that no smaller $s$ can be chosen, consider $N=2^{n}-1, A=B=2^{k} N$ and $s=n+2 k-1$. In this case $-2^{2 k} N \quad\left(\bmod 2^{s}\right)=\left(2^{2 k}-2^{n+2 k}\right) \quad\left(\bmod 2^{s}\right)=$ $2^{2 k}$. Therefore,

$$
\begin{gathered}
N R M M^{s}\left(2^{k} N, 2^{k} N, N\right)=\frac{2^{2 k} N^{2}+N\left(-2^{2 k} N \quad\left(\bmod 2^{s}\right)\right)}{2^{s}}= \\
\frac{2^{2 k} N^{2}+N 2^{2 k}}{2^{s}}=\frac{2^{2 k} N(N+1)}{2^{s}}=2 N
\end{gathered}
$$

(c) We have

$$
\begin{gathered}
N \operatorname{RM}^{s}(A, 1, N) \leq \frac{2^{k} N+N\left(2^{s}-1\right)}{2^{s}}= \\
N+\frac{N}{2^{n}} \cdot \frac{1}{2^{k}}\left(1-\frac{1}{2^{k}}\right)<N+1
\end{gathered}
$$

It follows that $N R M M^{s}(A, 1, N)$ can be at most $N$.

Since $N$ is odd and $N R M M^{s}(A, 1, N) \equiv A 2^{-s} \quad(\bmod N)$ equality to $N$ can occur only if $N$ divides $A$.

(d) Suppose that $S<\left(1+2^{k}\right) N$ before step 1.1 (note that $S$ is initialized to $0)$. Then, after step 1.1 we have $S<\left(1+2^{k}\right) N+B \leq\left(1+2^{k}\right) N+2^{k} N$. After step 1.2 we have $S<\left(\left(1+2^{k}\right) N+2^{k} N\right)+N=2\left(1+2^{k}\right) N$, and after step 1.3, we have again $S<\left(1+2^{k}\right) N$. Therefore, the maximal possible value for $S$ is $2\left(1+2^{k}\right) N$, which can be stored in $n+k+2$ bits.

Before demonstrating applications of Lemma 1, with different values of $k \geq 1$ and appropriate $s$, we start with the value $k=0$. This choice actually deals with the case $A, B<N$ (we ignore equality to $N$ ) and $s=n$, i.e., it reduces to the parameters range of the classical Montgomery multiplication. Here, Lemma 1 gives another explanation to the fact that in the computation of $\operatorname{MMUL}(A, B)$ (Algorithm 2), a single reduction step (2.4) is sufficient to reduce $S$ to the range $[0, N)$.

\section{Modular Exponentiation via the $N R M M^{s}$ Method}

Taking $k=1$ in Lemma 1, shows that modular exponentiation can be carried out by choosing $s=n+2$. We start with Algorithm 4 to describe the procedure.

Algorithm 4: (Modular Exponentiation via the $N R M M^{s}$ method) Input: $A, E, N(A<N, N$ is odd $), n=1+\left[\log _{2} N\right], m=1+\left[\log _{2} E\right], s=$ 
$n+2=3+\left[\log _{2} N\right], H=2^{2 s} \quad(\bmod N)$

Output: $A^{E}(\bmod N)$

Initialization: $A^{\prime}=N R M M^{s}(A, H, N) ; T_{m-1}=A^{\prime}$

$S=0$

For $i$ from $m-2$ to 0 do

$4.1 \quad T_{i}=N R M M^{s}\left(T_{i+1}, T_{i+1}, N\right)$

$4.2 \quad$ if $E_{i}=1$ then $T_{i}=N R M M^{s}\left(T_{i}, A^{\prime}, N\right)$

End For

$4.3 T_{0}=N R M M^{s}\left(T_{0}, 1, N\right)$

Return $T_{0}$

The correctness of Algorithm 4 follows from Lemma 1 (using $k=1$ ). We show that all inputs to $N R M M^{s}$ are legal, that is less than $2 N$. Since $A<N<2 N$, it is a legal input to $N R M M^{s}$ (that is, to the initialization step of Algorithm 4), and the resulting $A^{\prime}$ satisfies $A^{\prime}<2 N$. Consequently, $A^{\prime}$ is also a legal input to $N R M M^{s}$ (step 4.2). For the same reason, $T_{m-1}<2 N$, implying that $T_{i}<2 N$ for all $i$, and thus $T_{i}$ is a legal input as well. Finally, after the transformation back to the real domain (step 4.3) $T_{0}<N$. We remark that technically, we have only $T_{0} \leq N$, but in practice, the inequality is strict. The reason is that we typically choose $N$ to be either a prime or the product of two primes. Since $A<N$ to begin with $T_{0}$ cannot be a multiple of $N$ in any step of Algorithm 4, and thus, we end up with $T_{0}<N$.

Example. The following example demonstrates the use of the Algorithm 4, shows that nonreduced intermediate results indeed appear in the calculations, and shows that the bound on the maximal bit-length of $S$ is tight.

We compute here $A^{E}(\bmod N)$ with $A=212, E=240\left(=[11110000]_{2}\right)$, $N=249$, namely $212^{240} \quad(\bmod 249)=241$.

Here, $m=1+\left[\log _{2} E\right]=8$ and $n=1+\left[\log _{2} N\right]=8$. We use $s=n+2=10$. $H=2^{2 s}(\bmod N)=2^{20}(\bmod 249)=37$. Pre-computation gives $A^{\prime}=$ $N R M M^{s}(A, H, N)=209\left(=T_{7}\right.$ in Algorithm 4). The steps of the square and multiply algorithm are given in Table 1 . Column 1 shows the index $i$ (in Algorithm 4) running from $i=m-2=6$ down to $i=0$. Column 2 shows the relevant bit $\left(E_{i}\right)$ of the exponent. Columns 3 , and 4 show the inputs to steps 4.1, 4.2, and column 5 holds the result after the square and (conditional) multiply are performed. This value is fed into the subsequent step.

The final exponentiation result is obtained (step 4.3) by back transforming $T_{0}$ to the real domain, i.e., by computing $T_{0}=N R M M^{s}\left(T_{0}, 1, N\right)=$ $\operatorname{NRMM}^{s}(25,1,249)=241$.

Note that some results of the intermediate $N R M M^{s}$ steps are not reduced: step 4.2 in iterations $i=6,5$, and 4 , and step 4.1 in iteration $i=3$. However, these nonreduced results (output of $N R M M^{s}$ ) never exceed $2 N$ (= 498 in our case), and are therefore legal input to a subsequent $N R M M^{s}$ operation.

Table 2 shows the details of the calculation of $N R M M^{s}$ in iteration $i=3$ of Table 1, namely the calculation of $N R M M^{10}(319,319,249)$. 
Table 1. Computing $212^{240}(\bmod 249)$. Details are given in the text

\begin{tabular}{|c|c|c|c|c|}
\hline$i$ & $E_{i}$ & $T_{i+1}$ & $T_{i+1}$ & $T_{i}$ \\
\hline 6 & 1 & 209 & 235 & 269 \\
\hline 5 & 1 & 269 & 121 & 254 \\
\hline 4 & 1 & 254 & 241 & 296 \\
\hline 3 & 0 & 296 & 319 & 319 \\
\hline 2 & 0 & 319 & 175 & 175 \\
\hline 1 & 0 & 175 & 160 & 160 \\
\hline 0 & 0 & 160 & 25 & 25 \\
\hline
\end{tabular}

Table 2. Computing $N R M M^{10}(319,319,249)$. Details are given in the text

\begin{tabular}{|c|c|c|c|c|c|}
\hline$i$ & $A_{i}$ & $S=S+A_{i} B$ & $S_{0}$ & $S=S+S_{0} N$ & $S=S / 2$ \\
\hline 0 & 1 & 319 & 1 & 568 & 284 \\
\hline 1 & 1 & 603 & 1 & 852 & 426 \\
\hline 2 & 1 & 745 & 1 & 994 & 497 \\
\hline 3 & 1 & 816 & 0 & 816 & 408 \\
\hline 4 & 1 & 727 & 1 & 976 & 488 \\
\hline 5 & 1 & 807 & 1 & 1056 & 528 \\
\hline 6 & 0 & 528 & 0 & 528 & 264 \\
\hline 7 & 0 & 264 & 0 & 264 & 132 \\
\hline 8 & 1 & 451 & 1 & 700 & 350 \\
\hline 9 & 0 & 350 & 0 & 350 & 175 \\
\hline
\end{tabular}

Here, $A=B=319=[100111111]_{2}$. The result, 175 , is reduced in this case. As Lemma 1 predicts indeed, $S<6 N=1494$ in all steps. However, note that in step $i=5, S=S+S_{0} N=1056>2^{10}$. This shows that the bound given in Lemma 1 (d) is sharp: for $k=1$, the accumulator must hold $n+3$ bits $(=11$ in this example), and this number of bits cannot be decreased.

We point out that attempting to use $s<n+2$ in the exponentiation process may fail. With the input under consideration, using $s=n+1=9$ gives the correct result, but if we choose $s=n=8$, we get 296428706871896110475206 . Modulo $N$, this number is, of course, 241, and this overflow illustrates exactly the danger of choosing an inappropriate $s$.

For the sake of completeness, we give an example where the choice $s=n+1$ is not sufficient to assure that the output is bounded by $2 N$. Consider $N=255$, $A=505, B=507, n=1+\left[\log _{2} 255\right]=8, s=n+1=9$. Computation shows that $N R M M^{s}(A, B, N)=645>2 N(=510)$.

\section{Choosing $s$ for a Chain of Operations in $\mathbb{Z}_{N}$}

We have already showed that exponentiation requires the choice of $s=n+2$. The reason for the sufficiency of this relatively small value is that the "square and 
multiply" exponentiation algorithm involves only multiplications (and squaring), and no other operations in $\mathbb{Z}_{N}$. When other operations are involved, a higher value of $s$ may be necessary. The following proposition shows that there always exists a suitable value of $s$, with which the operations can be performed in the Montgomery domain, using $N R M M^{s}$ instead of modular multiplication.

\section{Proposition 1.}

Let $N$ be a given positive odd number, and $n=1+\left[\log _{2} N\right]$. Let $\left\{A_{j}, j=\right.$ $1,2, \ldots, r\}$ be a set of $r$ inputs (positive integers), such that for all $j, A_{j} \leq 2^{d} N$ for some $d \geq 0$.

Suppose that a sequence of operations in $\mathbb{Z}_{N}$ (modular addition, modular subtraction, modular multiplication) is to be performed, where the inputs to each operation are either taken from $\left\{A_{j}\right\}$, or are the results of a previous operation in the sequence.

Then, there exists a value of $s$ with which the computation can be performed in the following way:

1. Convert the inputs to the Montgomery domain, i.e., set $A_{j}^{\prime}=N R M M^{s}\left(A_{j}, H, N\right), j=1,2, \ldots, r$, where $H=2^{2 s} \quad(\bmod N)$.

2. Replace each modular multiplication in the sequence by an NRMM $M^{\text {s }}$ operation. Replace each modular addition by regular addition. Replace each modular subtraction, $X-Y$, by $2^{l} N+X-Y$ for $l$ such that $2^{l} N+X-Y \geq 0$.

3. Perform the modified sequence of operations, using the converted inputs.

4. Convert the desired result, $R$, to the real domain by $\operatorname{NRM}^{s}(R, 1, N)$.

Proof. We start with some $s \geq n+2 d$, baring in mind that the final value of $s$ is yet to be determined. Suppose that all the inputs are already converted to the Montgomery domain by the operation $A_{j}^{\prime}=N R M M^{s}\left(A_{j}, H, N\right)$. Note that the choice $s \geq n+2 d$ guarantees that $A_{j}^{\prime} \leq 2 N$.

We now replace each modular addition and modular subtraction operation by plain addition $(A D D)$ and plain subtraction $(S U B)$. At this point we note that negative results may appear as a result of subtraction. Thus, to avoid dealing with negative integers, we modify all the $S U B$ operations in the following way: instead of $S U B(X, Y)=X-Y$ where the inputs are bounded by $X, Y \leq 2^{l} N$ for some $l$, we use $S U B l N(X, Y)=2^{l} N+X-Y=S U B\left(A D D\left(2^{l} N, X\right), \bar{Y}\right)$. In this case, $0 \leq S U B l N(X, Y) \leq 2^{l+1} N$. Of course, the result remains unchanged modulo $N$.

To assess the upper bound of the intermediate addition results, note that if $X, Y \leq 2^{l} N$ then $A D D(X, Y)=X+Y \leq 2^{l+1} N$.

We now show how to determine an appropriate value of $s$. Consider the modified sequence of operations, with the additional operation $\operatorname{NRMM}(\cdot, 1, N)$ appended to the end of the sequence (used for transforming the result back to the real domain). Suppose that the $i$-th operation is $N R M M^{s}\left(X_{i}, Y_{i}, N\right)$ where the input satisfies $X_{i}, Y_{i} \leq 2^{k_{i}} N$ for some $k_{i}$. In general, $k_{i} \geq d$ because the input can involve intermediate results obtained by previous additions/subtractions. Any choice of $s \geq n+2 k_{i}$ is appropriate at this point: by Lemma 1, this assures that the input to the $N R M M^{s}$ is legal, and also assures that the output is bounded by $2 N$. 
It now follows that we can set $s=n+2\left(\max _{i} k_{i}\right)$, where the maximum is taken over all occurrences of $N R M M^{s}$ in the sequence. This value is appropriate throughout the sequence of operations. In particular, it assures that the returned result is either reduced or equals $N$. We finally comment that the bound on $s$, computed here, is not necessarily tight, and that there may be other ways to work out a chain of operations.

The following example shows how Proposition 1 can be used for point doubling on an elliptic curve over $G F(p)$.

Example: point doubling on an elliptic curve over $\boldsymbol{G F}(\boldsymbol{p})$. Consider the elliptic curve $y^{2}=x^{3}+a x+b$ over $G F(p)$, where $p$ is an odd prime. Suppose that the curve is represented in projective coordinates. If the point to double is $\left(x_{1}, y_{1}, z_{1}\right)$, and we denote $2\left(x_{1}, y_{1}, z_{1}\right)=\left(x_{2}, y_{2}, z_{2}\right)$ we have

$$
\begin{gathered}
\lambda_{1}=3 x_{1}^{2}+a z_{1}^{4}, \quad z_{2}=2 y_{1} z_{1}, \quad \lambda_{2}=4 x_{1} y_{1}^{2}, \\
x_{2}=\lambda_{1}^{2}-2 \lambda_{2}, \quad \lambda_{3}=8 y_{1}^{4}, \quad y_{2}=\lambda_{1}\left(\lambda_{2}-x_{2}\right)-\lambda_{3} .
\end{gathered}
$$

This computation can be implemented in several ways. One method is the following sequence of 18 steps consisting of $5 A D D, 3 S U B l p$ (with $l=1,2$ or 3 ), and $10 N R M M^{s}$ operations.

In cryptographic applications, point doubling is part of a scalar multiplication process which involves repeated instances of point addition and point doubling. In this context, conversion to the Montgomery domain is performed only at the beginning, and conversion back to the real domain is performed only at the end of the computations. For brevity, we suppose here that the input $x_{1}, y_{1}, z_{1}$ and $a$ are already given in the Montgomery domain, and in the first time doubling is performed, they are bounded by $2 p$. The result we return is also given in the same Montgomery domain (we do not transform back to the real domain). The output of the doubling procedure satisfies $x_{2}<8 p, y_{2}<4 p$ and $z_{2}<2 p$. As explained below, this assures that this result can be reused as input for a subsequent doubling (or addition) procedure. The sequence of operations (in the Montgomery domain) is the following:

Compute the Montgomery base analog of $\lambda_{1}=a z_{1}^{4}+3 x_{1}^{2}$

1. $R 0=N R M M^{s}\left(z_{1}, z_{1}, p\right)$

2. $\quad R 0=N R M M^{s}(R 0, R 0, p)$

3. $R 0=N R M M^{s}(a, R 0, p)$

4. $T 1=N R M M^{s}\left(x_{1}, x_{1}, p\right)$

5. $T 2=A D D(T 1, T 1)$

6. $T 1=A D D(T 2, T 1)$

7. $T 1=A D D(R 0, T 1)$

Compute the Montgomery base analog of $z_{2}=2 y_{1} z_{1}$

8. $T 2=A D D\left(y_{1}, y_{1}\right)$

9. $z_{2}=N R M M^{s}\left(T 2, z_{1}, p\right)$

Compute the Montgomery base analog of $\lambda_{2}=4 x_{1} y_{1}^{2}$ 
10. $R 0=N R M M^{s}(T 2, T 2, p)$

11. $T 2=N R M M^{s}\left(x_{1}, R 0, p\right)$

Compute the Montgomery base analog of $x_{2}=\lambda_{1}^{2}-2 \lambda_{2}$

12. $T 3=N R M M^{s}(T 1, T 1, p)$

13. $T 4=A D D(T 2, T 2)$

14. $x_{2}=S U B 2 p(T 3, T 4, p)$

Compute the Montgomery base analog of $\lambda_{3}=8 y_{1}^{4}$

15. $T 3=N R M M^{s+1}(R 0, R 0, p)$

Compute the Montgomery base analog of $y_{2}=\lambda_{1}\left(\lambda_{2}-x_{2}\right)-\lambda_{3}$

16. $T 2=\operatorname{SUB} 3 p\left(T 2, x_{2}, p\right)$

17. $T 1=N R M M^{s}(T 2, T 1, p)$

18. $y_{2}=S U B 1 p(T 1, T 3, p)$

To determine the required value of $s$, we look at consecutive $A D D / S U B l p$ operations, and set $s$ to be high enough to assure that with the relevant input, the output of $N R M M^{s}$ is bounded by $2 p$.

After step 5 we have $T 2<4 p$, after step $6 T 1<6 p$, and after step $7 T 1<8 p$. In step 8 we have $T 2<4 p$, and thus for the $N R M M^{s}$ in step 9 we have $k_{1}=2$. The output $z_{2}$ is bounded by $2 p$. Steps 10 and 11 require $k_{2}=1$. Step 12 requires $k_{3}=3$. In step 13 we have $T 4<4 p$, and this is why $S U B 2 p$ is used in step 14. Note that $x_{2}=S U B 2 p(T 3, T 4, p)<6 p<8 p$. In step 15 we square $\left(4 y_{1}^{2}\right)^{2}=16 y_{1}^{2}$ and divide by 2 (to get $8 y_{1}^{2}$ ) by using $N R M M^{s+1}$. In step 15 we have $T 2<2 p$ (from step 11) and $x_{2}<8 p$, and this is why we use $S U B 3 p$ in step 16. Therefore, for the $N R M M^{s}$ operation in step 17 we use $k_{4}=4$. Finally, in step 18 we compute the output $y_{2}$ which is bounded by $4 p$.

Consequently, the value $s=n+2 \max k_{i}=n+8$, where $n=1+\left[\log _{2} p\right]$, is an appropriate choice.

In the above procedure we assure that $x_{2}<8 p, y_{2}<4 p$ and $z_{2}<2 p$. With our choice of $s$, these values are proper inputs for a subsequent point doubling (i.e., can the roles of $x_{1}, y_{1}, z_{1}$ ). This can be verified by noting that $x_{1}, z_{1}$ are used in $N R M M^{s}$ operation (steps $1,4,11$ ), and $y_{1}$ is used in step 8, in an $A D D$ operation. This operation yields $T 2$, which is then a legal input to the $N R M M^{s}$ operation in step 9. Although we do not give here the details we point out that $x_{2}<8 p, y_{2}<4 p$ and $z_{2}<2 p$ are legal inputs to the procedure implementing point addition as well.

\section{Discussion}

Proper choice of the parameter $s$ enables using the $N R M M^{s}$ in a variety of applications and contexts, while keeping modular reduction not required in any stage. Consequently, using $N R M M^{s}$ in hardware implementations can save on time and/or hardware requirements. Another important feature of $N R M M^{s}$ (i.e., of Algorithm 1) is in being homogeneous: its execution does not depend on the input (in contrast with Algorithm 2). Thus, timing attacks as well as other 
attacks to which the classical Montgomery multiplication is susceptible [3] do not affect systems using the $N R M M^{s}$.

We further comment that there may be different ways to implement a given sequence of operations in $\mathbb{Z}_{n}$, and the flexibility in choosing $s$ may help in optimizing the computations in various contexts.

Finally, we mention that Lemma 1 can be generalized to the situation where $A \leq 2^{\alpha_{1}} N, B \leq 2^{\alpha_{2}} N \alpha_{1}, \alpha_{2} \geq 0$, and the result of $N R M M^{s}$ is to be smaller than $2^{\alpha_{3}} N$ for some $\alpha_{3} \geq 1$. In such cases, we need to choose $s=n+\alpha_{1}+\alpha_{2}+$ $1-\alpha_{3}$. Proof and applications where different values of $\alpha_{1}, \alpha_{2}, \alpha_{3}$ are useful, will be discussed in subsequent publication.

Acknowledgments. I thank Or Zuk for helpful discussions, suggestions, and help in preparing the paper.

\section{References}

1. Blum, T., Paar, C.: Montgomery modular exponentiation on reconfigurable hardware. In Proceedings of the 14th symposium on computer arithmetic, (1999) 70-77

2. Montgomery, P. L.: Modular multiplication without trial division. Mathematics of Computation 44 (1985) 519-521

3. Schindler, F.: A timing attack against RSA with Chinese Remainder Theorem. Lecture Notes Comp. Sci. 1965 (2000) 110-124

4. Walter, C. D.: Montgomery exponentiation needs no final subtractions. Electronics Letters 35 (1992) 1831-1832 